theoretical and semi-empirical conformational methods. Each lecture has from 9 to 61 pages and lists from 6 to 82 references. A subject index follows after the papers. Since many chapters are comprehensive, this book will be suitable for the education of graduate students and junior scientists of molecular biology and biophysics. Furthermore, the references will be valuable for scientists who have an interest in these fields.

All the papers are printed directly from masters provided (as typescripts) by the authors; the reader should be aware that some mistakes and imperfections of description could not be avoided in this book.

The lectures have been classified into three sections, on the basis of the methods involved.

The title of the first section is $X$-ray diffraction and related methods. In this section, the first three chapters are related to methods for single crystals, the following two are related to the analytical method for protein structure, and the last two chapters deal with the analytical method for fibrous materials and the structure of viruses, respectively. The first chapter, which is on the determination of heavy-atom sites and which provides a checklist of the most popular methods, is the least satisfactory of the book: it is too difficult for a newcomer to understand because there is so little description of the basic principles. At least the formulae in which each coefficient in Table 1 would be used should have been given by the author - otherwise, one of his conclusions regarding the 'hand of heavy-atom sites' must be reversed, if we apply his coefficient $\left(\Delta F_{\text {ano }}^{l}, \alpha_{p}+90^{\circ}\right)$ to one of the most popular Fourier summations. The sample maps which were illustrated, using simulated $5 \mathrm{~A}$ data, might be helpful to some students but they have no practical meaning because they are so far removed from real maps. The next chapter, by Hendrickson, describes phase evaluation in macromolecular crystallography. This is one of the most important steps of protein structure analysis, and his description covers almost all the important points. The following chapter, by Diamond, gives the principle and properties of the method of least squares. For refinement, this method is powerful and is a widely used technique. In this chapter the treatment is very comprehensive and diagrams are employed to assist the clarity of the explanation. A chapter by Srinivasan describes protein structural data presentation: Srinivasan represents protein structure by using a combination of parameters of distances or angles. This analysis will be valuable for finding the general features in protein structure. The chapter by Schulz describes the structural organization of globular protein on the basis of the regularity found in the three-dimensional structure. Schulz's hierarchy is simple, and we were able to read through it very easily. The last chapter of this section deals with the structure of simple viruses by electron-microscope and X-ray methods. The description of rod-shaped viruses, especially TMV, from a historical viewpoint, is interesting and is illustrated with many figures; but there are only a few diagrams in the description of the structure of spherical viruses. Anyhow, the reader should take note that the progress of this field is rapid and the latest references given for this chapter are dated 1978.

The title of the next section is Optical and spectroscopic methods. Four chapters deal with fluoroescence spectroscopy, circular dichroism (CD), Raman, and NMR spectroscopy. The first chapter, by Cantor, describes the dynamic aspect of fluorescence spectroscopy. Cantor gives a simple explanation of the principles and experimental method, and describes three specialized applications: environmental sensitivity, anisotropic polarization and singlet-singlet energy transfer. The feature of this method is that the behaviour of one molecule can be observed in a given time scale; but the application to macromolecules still has some limitations. The next chapter is on the application of CD to the study of polynucleotides and nucleic acid, and the following chapter deals with the application of Raman spectroscopy to nucleic acid. The last chapter, by Wüthrich, gives high-resolution NMR studies of peptides and proteins. NMR has recently become an attractive technique for the investigation of molecular conformation in solution. Wüthrich describes the features of ${ }^{1} \mathrm{H},{ }^{13} \mathrm{C}$ and ${ }^{15} \mathrm{~N}$ spectra and the elucidation of structure and conformation by NMR. This chapter will be interesting for protein crystallographers who are working on structure refinement with high-resolution data.

The title of the last section is Theoretical and semiempirical methods of conformational analysis. The first two chapters discuss the conformational structures of polynucleotides and polysaccharides respectively. The latter chapter, by Rao, contains many of the author's own results, obtained by using a semi-empirical potential function. The title of the next chapter, by Nagano, is Seeming algorithms of protein folding. This is a consideration of long-range interactions, and the theoretical basis of the prediction of structure derives from the careful inspection of all known tertiary structures. Thus, this treatment sometimes contains ambiguous and arbitrary choices of assumption. The last chapter gives the structure and conformational analysis of gastric mucus gels by biophysical and chemical methods.

Finally, we must express gratitude to the publishers for having produced this interesting book at such a low price that everyone can buy a copy.

$\begin{array}{ll}\text { Department of Chemistry } & \text { K. SAKABE } \\ \text { Nagoya University } & \text { N. SAKABE } \\ \text { Chikusa } & \\ \text { Nagoya } 464 & \\ \text { Japan } & \end{array}$

Acta Cryst. (1983). B39, 143

Phasendiagramme. By P. PAufler. Pp. 177. Berlin: Akademie Verlag, 1981. Price DM 8.00.

This book presents a well arranged summary of the science of phase diagrams of heterogeneous systems.

It is divided into chapters describing: basic phenomena; principles of equilibria in the thermodynamic system; phase equilibria in one-, two- and three-component systems; and analysis and representation of phase equilibria in four and more component systems. The connection between the atomic architecture of matter and the macroscopic thermodynamic functions is briefly summarized.

The analytical description of thermodynamic principles is appropriately exemplified by graphical representation and by discussion of the most common phase diagrams - mostly from the field of metallurgy, but some belonging to salt and oxide systems.

The book is eminently readable. Numerous references are given to sources which treat particular problems more thoroughly. 
The book has definite didactic value. It can be recommended for graduate study, but those working in the fields of chemistry, physics, materials science, etc., will also find it useful (and pleasant) reading.

J. MAJLING

Department of Chemical Technology of Silicates

Faculty of Chemical Technology

Slovak Technical University

Janska 1

88037 Bratislava

Czechoslovakia

Acta Cryst. (1983). B39, 144

\section{Books Received}

The following books have been received by the Editor. Brief and generally uncritical notices are given of works of marginal crystallographic interest; occasionally a book of fundamental interest is included under this heading because of difficulty of finding a suitable reviewer without great delay.

Crystallography. By R. Steadman. Pp. viii + 120. Wokingham. England: Van Nostrand, 1982. Price £3.95. A review of this book, by $\mathrm{H}$. Mendel, has been published in the January 1983 issue of Acta Cryst. Section A, p. 190.

Physics of intercalation compounds. Edited by L. PIETRoNERo and E. TosatTI. Pp. ix + 323. Berlin, Heidelberg: Springer-Verlag, 1981. Price DM 60, US \$28. A review of this book, by G. S. Parry, has been published in the January 1983 issue of Acta Cryst. Section A, pp. 188-189.

The spindle stage: principles and practice. By F, DonALD Bloss. Pp. xii + 340. Cambridge University Press, 1981. $15 \times 23 \mathrm{~cm}$. Hard covers. Price UK $£ 35.00$ (net). A review of this book, by Gabrielle and J. D. H. Donnay, has been published in the January 1983 issue of Acta Cryst. Section A, pp. 189-190. 